\title{
Salz, eine Brezel und der Weg zum Bahnhof. Strategien des Bittens in Varietäten des Deutschen und im Serbischen
}

Elke Hentschel (Bern)

\begin{abstract}
This paper examines the verbal strategies used by speakers of German in Germany and Switzerland and speakers of Serbian in Serbia in order to voice a request. The participants in the study were asked what they would say in the following three situations: Asking for the way to the railway station in a strange city, asking their younger brother to pass the salt at the family dinner table, and buying a pretzel at the local bakery. Subsequently, the use of downtoners like 'please' or special particles was analysed, as well as the frequency of non-indicative verbal modes (subjunctive or conditional), the occurrence an equivalent of excuse me at the beginning of the request, and the use of greetings and address forms. The results show surprising differences between the three groups.
\end{abstract}

\section{$1 \quad$ Einleitung}

Anlass für die kleine Untersuchung, aus der im Folgenden eine Auswahl von Ergebnissen vorgestellt werden sollen, war ein in mehreren Schweizer Tageszeitungen publiziertes Interview mit Christoph Stokar, Autor des 2012 erschienenen Buches Der Schweizer Knigge. Was gilt heute? Auf die Journalistenfrage: "Sie haben einen Knigge speziell für Schweizer geschrieben. Sind wir so anders als etwa die Deutschen?" antwortete er:

Nur schon unsere Sprache ist viel höflicher. Wir brauchen den Konjunktiv: «Dürfte ich bitte einen Kaffee haben?» In Deutschland heisst es: «Einen Kaffee bitte!» Wir funktionieren auch nicht so hierarchisch. Wir sind eine Willensnation und wünschen uns eine Gesellschaft im Gleichgewicht. Was die Harmonie stört, mögen wir nicht. In Deutschland biedert sich der Chef gegen oben an und tritt gegen unten.

(Tages Anzeiger, 14.12.2012)

Aus diesen Ausführungen könnte man nunmehr beispielsweise die folgenden Schlussfolgerungen ziehen:

1. Konjunktive sind höflich.

2. Angehörige einiger Nationalitäten sind höflicher als andere.

3. Mangel an Höflichkeit geht mit negativen Charaktereigenschaften und den ihnen entsprechenden Verhaltensweisen (etwa: sich nach oben anbiedern, nach unten treten) einher.

Linguistik online 72, 3/15 - http://dx.doi.org/10.13092/lo.72.1971

CC by 3.0 
Warum ausgerechnet ein unhöflicher Mensch sich gegenüber einigen Kommunikationspartnern anbiedern und sich somit also nicht etwa unhöflich, sondern im Gegenteil übertrieben höflich und zuvorkommend verhalten sollte, ist nicht auf Anhieb verständlich; und auch der Zusammenhang zwischen einer Kaffeebestellung mit bitte, jedoch ohne Konjunktiv, und den Charaktereigenschaften der bestellenden Person ist nicht ohne Weiteres ersichtlich. Abgesehen von solchen Detailüberlegungen ist die zentrale Frage hier aber natürlich: Wie kommt jemand dazu, überhaupt eine solche Wertung, verbunden mit einer Zuordnung nach Nationalität, vorzunehmen?

Die Beobachtung, dass Menschen nicht überall auf der Welt dieselben sprachlichen Handlungen auf dieselbe Art und Weise ausführen und also auch nicht unbedingt mit genau derselben Formulierung Kaffee bestellen, ist per se natürlich trivial. Von den komplexen pragmatischen Regeln, die in verschiedenen Sprachgemeinschaften gelten, einmal abgesehen, hindert allein schon die Tatsache, dass nicht alle Sprachen über einen Konjunktiv verfügen, Mitglieder der entsprechenden Sprachgemeinschaft daran, diese Form zu Höflichkeitszwecken zu verwenden. Dass es hingegen wirklich irgendwo auf der Welt eine Gesellschaft gibt, deren Mitglieder nach Maßgabe ihrer eigenen Regeln stets unhöflich zueinander sind - also etwa so, als würden Muttersprachler des Deutschen die Bedienung im Café regelmäßig mit Kaffee her, aber dalli! anblaffen -, wird vermutlich niemand ernsthaft annehmen. Dennoch kann die Wahrnehmung von Unterschieden zwischen den pragmatischen Regeln verschiedener Gruppen offenkundig dazu führen, dass wie im oben zitierten Fall ganzen Sprachgemeinschaften mangelnde Höflichkeit und ein mieser Charakter zugeschrieben wird: Man misst die „fremde“ Pragmatik mit den Maßstäben der eigenen und kommt zu dem Schluss, dass sie unzureichend ist. Dass man die Äußerung Dürfte ich bitte einen Kaffee haben? erwartet, könnte also zumindest erklären, warum jemand Einen Kaffee, bitte! für ein Anzeichen mangelnder Höflichkeit hält. Auch dies wäre allerdings nicht weiter bemerkenswert. Interessanter ist hingegen die Frage: Sind die gruppenspezifischen Regeln wirklich so, wie sie hier angesetzt werden - oder verbergen sich in Wirklichkeit nationale Stereotype und Vorurteile hinter der Annahme, die gar nicht der Realität entsprechen? Immerhin wäre es mit den Forschungsergebnissen zu interkulturellen Stereotypen gut vereinbar (vgl. z. B. Thomas 2006 sowie die dort angeführte Literatur), eine gegen die Außengruppe gerichtete kognitive Konstruktion anzunehmen, die dann mit der Wirklichkeit gar nichts zu tun haben muss.

Um festzustellen, ob in den verschiedenen Sprachgruppen wirklich unterschiedliche Regeln angewandt werden, die zu solchen Stereotypisierungen führen könnten, wurde eine kleine empirische Untersuchung mit deutschen, Schweizer und serbischen Sprechern durchgeführt, deren Ergebnisse im Folgenden ausschnittsweise vorgestellt werden sollen. Die letztgenannte Gruppe dient dabei in gewisser Hinsicht als Tertium comparationis, indem sie sich sowohl hinsichtlich Sprache als auch Kultur von den beiden anderen unterscheidet.

\section{Grundlagen}

Höflichkeit - im Sinne von konkretem höflichem Verhalten - wird in der aktuellen Forschungsliteratur durchweg als soziales Konstrukt gesehen, als Teil des Diskurses einer Gesellschaft, der damit naturgemäß keinen Anspruch auf Allgemeingültigkeit erheben kann (cf. z. B. Watts 2003: 27-46). Als zentrales Konzept auf dem Gebiet der Höflichkeitsfor- 
schung, die mittlerweile eine kaum noch überblickbare Vielzahl von Publikation hervorgebracht hat, kann die von Brown und Levinson (1987/ $\left.{ }^{18} 2008\right)$ in die Diskussion eingebrachte, ihrerseits auf Goffman (1955/2005) zurückgehende Annahme eines sozialen Gesichts (face) angesehen werden, das bei ihnen in ein positives und ein negatives Gesicht unterteilt wird. Dieses Modell, aus dem dann eine positive und eine negative Höflichkeit abgeleitet wird, gilt in vieler Hinsicht nach wie vor als grundlegend (cf. z. B. Leech 2014: 113, bei ihm: "pospoliteness" und "neg-politeness"), auch wenn es in den Folgejahren Kritik erfahren hat.

Während Brown/Levinson (1987// 2008$)$ Höflichkeit als Mittel zur Vermeidung von gesichtsbedrohenden Akten ansehen, betrachten sie andere eher als Mittel zur Vermeidung von Reibungen oder zur gegenseitigen Unterstützung und zum Erzeugen von Wohlbefinden (cf. Watts 2003: 49-53). Einen Überblick über die Geschichte und die aktuelle Entwicklung der Höflichkeitsforschung gibt Locher (2012), die darauf hinweist, dass sich in jüngerer Zeit einerseits ein zusätzliches Interesse am Gegenteil von Höflichkeit, an "impolite and rude behaviour" (ibd.: 44) entwickelt hat und dass andererseits zunehmend auch umfassendere Forschungsbereiche wie soziale Kognition und Identitätsforschung mit einbezogen werden.

Auffallend bei näherer Betrachtung der Höflichkeitsforschung ist, dass es offenbar zwei diametral entgegengesetzte Ziele gibt, die sich in der einen oder anderen Formulierung zur Definition von Höflichkeit regelmäßig wiederfinden: auf der einen Seite das Vermeiden von Schaden (Gesichtsverlust, Reibungen), auf der anderen das Erzeugen eines Nutzens (Unterstützung, Wohlbefinden). Diese Dichotomie erinnert stark an Schopenhauers (1851) Parabel von den Stachelschweinen, die sich im Winter aneinander wärmen wollen, ohne sich gegenseitig mit ihren Stacheln zu verletzen, und dabei auf der Suche nach dem optimalen Abstand sind, der möglichst viel Wärme mit möglichst wenig Stacheln vereint. Auch menschliche Gesellschaften funktionieren nach Schopenhauers Ansicht so, und "die mittlere Entfernung, die sie endlich herausfinden und bey welcher ein Zusammenseyn bestehn kann, ist die Höflichkeit und feine Sitte" (ibd.: 525). Im Grunde genommen impliziert schon das face-Modell von Brown und Levinson ein solches doppeltes Ziel: nicht mit den Stacheln anderer Bekanntschaft machen zu wollen, entspräche dann dem negative face, während man das Bedürfnis nach Wärme mit dem positive face gleichsetzen könnte.

Für die Wahrnehmung einer konkreten Äußerung in einer realen Situation spielen die drei Faktoren Macht, Distanz und Grad der Zumutung oder Gesichtsbedrohung (social power, social distance und imposition in der Terminologie von Brown/Levinson 1987/2008: 74 et passim) eine zentrale Rolle. Sie sind in der Forschung inzwischen empirisch gut belegt und können insgesamt als hochgradig bestätigt angesehen werden (cf. hierzu z. B. EconomidouKogetsidis 2010: 2263-2265 und die dort angegebene Literatur). Holtgraves (2005: 82f.) erläutert ihr Zusammenspiel folgendermaßen:

If a speaker uses a very polite form, for example, will others infer that he or she is relatively low in status, that the act is very threatening, or that the relationship is a distant one? Sometimes information exists regarding one or two of the dimensions such that inferences will be most likely on the unspecified dimension. For example, a boss (high power) making a request to an employee (high distance) with a relatively polite form may implicate, through his high level of politeness, a view that the request is somewhat imposing.

Man kann sich diese Überlegungen leicht an alltäglichen Situationen verdeutlichen. So dürfte 
für die meisten Muttersprachler des Deutschen die Vorstellung absurd sein, dass jemand am Frühstückstisch seinen in die Zeitung vertieften Lebensgefährten oder seine Lebensgefährtin mit folgenden Worten um den Zucker bitten würde:

Entschuldige bitte vielmals, ich wollte nur fragen, ob du mir mal eben den Zucker geben könntest.

In Sie-Form im Café gegenüber einer fremden Person geäußert, die am Tisch nebenan in die Zeitung vertieft ist und hinter deren Ellenbogen für den Sprecher unerreichbar der Zucker steht, wäre der Satz hingegen durchaus möglich, und er würde vermutlich auch als völlig angemessen wahrgenommen werden:

Entschuldigen Sie bitte vielmals, ich wollte nur fragen, ob Sie mir mal eben den Zucker geben könnten.

Aber nicht nur die gesellschaftliche Rolle bzw. das Machtgefälle zwischen den Kommunikationspartnern, die soziale Distanz und der Grad der Zumutung, den die Erfüllung der Bitte für die angesprochene Person impliziert, spielen für die Wortwahl eine Rolle. Dasselbe gilt, wie zahlreiche Studien bestätigen, für den kulturellen Rahmen, innerhalb dessen eine Äußerung getätigt wird und der für die verwendeten verbalen wie auch nonverbalen Signale ausschlaggebend ist (cf. z. B. Kim/Guan/Park 2012; Lee 2011; Nishijima 2014; Ogiermann 2009; $\mathrm{Zhu} / \mathrm{Bao}$ 2010). Es gibt insbesondere für Sprechakte wie Bitten oder Entschuldigungen Regeln und Normen, die von Sprache zu Sprache, aber auch innerhalb derselben Sprache von Region zu Region variieren können. Was in der einen Sprache und Region höflich ist, könnte daher in der anderen unpassend oder gar regelrecht unhöflich wirken. Dies könnte theoretisch die Grundlage für die eingangs zitierte Wahrnehmung einer Bevölkerungsgruppe als im Verhältnis zu einer anderen höflicher sein; und hier setzt die im Folgenden vorgestellte Studie an, die an alltäglichen Situationen mit eindeutig definierten sozialen Rollen - also unter Festschreibung von Machgefälle, Distanz und Grad der Zumutung - zu ergründen versucht, welche Formulierungen wo bevorzugt werden.

Aufforderungen sind grundsätzlich sehr gut dafür geeignet, sprachliche Strategien der Höflichkeit zu untersuchen: Eine Aufforderung ist unabhängig davon, ob man das theoretische Konzept face akzeptiert und wenn ja, wie man es gegebenenfalls genau definiert, stets ein Eingriff in den Handlungsbereich des Gegenübers, das damit im Normalfall ja zu einer Handlung gebracht werden soll, die ohne die Aufforderung nicht erfolgt wäre. Man kann infolgedessen erwarten, dass eine "nackte" Aufforderung, etwa in Form eines reinen Imperativs ohne jeglichen abmildernden Zusatz, nur in Ausnahmefällen verwendet würde - beispielsweise in einer Notsituation - und dass in anderen Fällen sprachliche Mittel zum Einsatz kommen, mit denen die Aufforderung abgemildert wird. Blum-Kulka/Olshtain (1986) haben für diese sprachlichen Strategien der Abmilderung bereits früh eine Skala der Indirektheit entwickelt, die auf den folgenden drei Ebenen basiert:

- die direkte, explizite Ebene, wie sie etwa durch die Wahl des Imperativs realisiert wird: Look out! Take cover! ${ }^{1}$

- die konventionalisierte indirekte Ebene, die sich indirekter Sprachakten bedient und sich

\footnotetext{
${ }^{1}$ Im Deutschen wird in solchen Situationen mehrheitlich ein Substantiv gebraucht: Vorsicht! Deckung! etc.
} 
typischerweise etwa im Gebrauch von Interrogativsätzen zeigt: Könnten Sie... Would you... - die nicht konventionalisierte indirekte Ebene, etwa Andeutungen oder indirekte Hinweise.

(cf. ibd.: 201)

Für das Deutsche wie für das Serbische, die beide über den verbalen Modus des Imperativs verfügen, bestünde eine explizite und direkte Aufforderung somit im Gebrauch der entsprechenden Verbform. In beiden Sprachen kann man ferner zusätzlich noch zwischen einer näheren $(d u / t i)$ und einer distanzierteren (Sie/vi) Anredeform wählen:

Komm her! Kommen Sie her!

vs.

Dodji ovamo! Dodjite ovamo!

Auf den ersten Blick scheinen auf der direkten und expliziten Ebene also in beiden Sprachen völlig parallele Verhältnisse zu herrschen. Dieser Eindruck ist indessen trügerisch. Denn während im Deutschen nur ein Verb mit zwei Personalformen zur Verfügung steht, bietet das Serbische aufgrund der Tatsache, dass es über zwei verschiedene Verbalaspekte verfügt, insgesamt vier Realisierungsmöglichkeiten an. Ein weiteres Imperativpaar zum Verb 'kommen' wäre im Serbischen das vom imperfektiven Verb dolaziti 'kommen' abgeleitete:

\section{Dolazi ovamo! Dolazite ovamo!}

Dabei unterscheiden sich die beiden Imperative dolazi(te) (imperfektiv) und dodji(te) (perfektiv) in pragmatischer Hinsicht beträchtlich. Um diesen Unterschied im Deutschen zum Ausdruck zu bringen, kann man den perfektiven Aspekt durch Hinzufügung der Modal- oder Abtönungspartikel ${ }^{2}$ mal zu übersetzen versuchen, den imperfektiven hingegen ohne einen solchen Zusatz (cf. hierzu z. B. Hentschel 1991; 2003: 60-61; 2013a: 66-67). Dann entspräche Dolazi ovamo in etwa dem deutschen Komm her!, Dodji ovamo! hingegen dem deutschen Komm mal her! und damit einer Form, die anders als die serbische Äußerung zusätzlich einen downtoner, ein Element der Abmilderung, beinhaltet. ${ }^{3}$

Dieses einfache Beispiel zeigt bereits, welche unerwarteten Schwierigkeiten sich hier beim Sprachvergleich ergeben können. Vor allem macht es deutlich, dass eine Aussage wie "In beiden Sprachen liegt ein Imperativ der Distanzform ohne weitere Zusätze vor" keineswegs ausreicht, solange man nicht sicherstellt, dass die strukturellen Bedingungen gleich sind und somit auch die verwendeten Formen wirklich als gleichwertig angesehen werden können. Dies ist hier nicht der Fall: Was in der einen Sprache mit einem zusätzlichen Element additiv ausgedrückt werden muss, ist in der anderen bereits in der Wahl des verbalen Aspekts enthalten und braucht keine zusätzlichen Ausdrucksmittel.

2 Die Begriffe "Abtönungspartikel" (zurückgehend auf Weydt 1969) und "Modalpartikel" bzw. "modale Partikel" (erstmals gebraucht bei Krivonosov 1963) werden hier gleichbedeutend und im Sinne dieser beiden Autoren verwendet.

${ }^{3}$ Interessanterweise wird bei Blum-Kulka/Olstain (1986: 204) im dort gegebenen deutschsprachigen Beispiel (40) Kannst du den Mist vielleicht mal eben wegräumen? nur vielleicht als "downtoner" eingeordnet. Dass daneben auch mal und eben verwendet werden, bleibt unbeachtet, und die Funktion dieser Zusätze wird nicht analysiert. 
Naturgemäß steht zu erwarten, dass Imperative in den meisten Fällen von abmildernden $\mathrm{Zu}$ sätzen begleitet werden, die ihre Direktheit zumindest teilweise abfedern sollen. Im Deutschen gehören dazu in erster Linie Modalpartikeln wie eben und doch (cf. Hentschel 2003: 61f.) und das bereits erwähnte mal sowie verschiedene Kombinationen daraus (cf. Komm doch mal eben her!). Vergleichbare Elemente im Serbischen wären časkom, začas (beides etwa: 'einen Augenblick'; 'mal eben') oder malo ('ein wenig'), also Äußerungen wie Dodji časkom/začas/malo ovamo! (cf. z. B. Bakšić 2012: 97). Selbstverständlich kann auch durch Hinzufügen von bitte oder dem entsprechenden serbischen Äquivalent molim eine weitere Abmilderung einer Aufforderung erfolgen (Komm doch mal bitte her! Dodji časkom ovamo, molim!) und man kann untersuchen, wie häufig und unter welchen Bedingungen das geschieht. Dennoch ist nochmals festzuhalten, dass bereits der Imperativ selbst in der einen Sprache nicht unbedingt dem in einer anderen gleichgesetzt werden kann, da hier in Sprachen mit Verbalaspekt Wahlmöglichkeiten bestehen, die andere Sprachen nicht kennen. So gesehen könnte man durchaus argumentieren, dass Formen wie Dodji časkom ovamo! gegenüber Komm mal her! einen downtoner mehr enthalten.

Anders als bei direkten und expliziten ist bei konventionalisierten indirekten Realisierungen von Aufforderungen von Vorneherein zu erwarten, dass es hier sehr viel mehr Spielraum im Bereich der Ausdrucksmöglichkeiten gibt und dass sie sich daher von Sprache zu Sprache sowie in Abhängigkeit von der Region auch innerhalb ein und derselben Sprache deutlich unterscheiden. Dennoch gibt es natürlich auch hier beobachtbare sprachübergreifende Gemeinsamkeiten, ${ }^{4}$ so etwa die, dass eine Aufforderung in Frageform gekleidet und mit einem Modalverb wie 'wollen' oder 'können' modifiziert wird. Man äußert dann wörtlich genommen keine Aufforderung, sondern fragt nur, ob das Gegenüber den Wunsch oder die Möglichkeit hat, etwas Bestimmtes zu tun. Dieser Mechanismus ist sehr typisch, und er dürfte vermutlich in der Mehrzahl der Sprachen zu finden sein. Auch hier können zusätzliche downtoner verschiedenster Art verwendet werden, beispielsweise modale Partikeln wie mal oder vergleichbare Elemente wie časkom, ferner Modalwörter wie vielleicht (serbisch: možda), spezifische Zusätze wie bitte (molim), einleitende entschuldigende Vorsätze wie entschuldigen Sie, verzeihen Sie (oprostite, izvinite) und vieles andere mehr. Abermals ist es interessant zu sehen, welche dieser verschiedenen Strategien bzw. welche Kombination daraus in einer Sprachgemeinschaft bevorzugt wird.

Völlig divergent wird die Ausdrucksform einer Bitte logischerweise dann, wenn es sich um den "nonconventional indirect level" (Blum-Kulka/Olshtain 1984: 201) handelt. Während indirekte Formen der Aufforderung durchaus konventionalisiert werden, ist hiermit nun wirklich eine Ebene angesprochen, in der die Aufforderung nur ganz verdeckt geäußert wird eben so, wie es erstmals bei Searle (1975) detailliert beschrieben wird. Das klassische Beispiel für diese Art des Sprechens ist der Hinweis auf Kälte oder Zugluft, durch den das Gegenüber dazu bewegt werden soll, das Fenster zu schließen. Hier ist ein Vergleich der gewähl-

\footnotetext{
${ }^{4}$ Gelegentlich findet sich die Auffassung, dass auch „,concepts such as 'imposition', 'politeness', 'tact', 'directness', 'face', 'relevance', 'interaction"' in Wirklichkeit sprach- bzw. kulturspezifisch, genauer: "anglozentrisch" sind (Goddard/Cliff 2014: 69). Für die in der vorliegenden Untersuchung betrachteten Sprach- und Kulturgemeinschaften soll jedoch unterstellt werden, dass sie über solche Konzepte verfügen und dass diese in der kommunikativen Praxis eine zentrale Rolle spielen.
} 
ten Form im Grunde nicht mehr möglich, und man kann nur grundsätzlich feststellen, ob zur Übermittlung der Bitte auf diese Äußerungsebene zurückgegriffen wird.

Aufforderungen können naturgemäß unter sehr unterschiedlichen Bedingungen und in verschiedenen Kontexten erfolgen, was sich nicht zuletzt auch im Lexem selbst spiegelt, mit dem sie bezeichnet werden. Bei eindeutigem Machtgefälle zugunsten der sprechenden Person bzw. bei einem Mangel an abweichenden Handlungsoptionen auf Seiten der angesprochenen Person (so die Definition eines Befehls bei Leech 2014: 134f.) wäre Befehl der passende Ausdruck; bei sozialer Gleichstellung oder wenn sich die Aufforderung umgekehrt an eine höhergestellte Person richtet bzw. wenn die angesprochene Person die Ausführung auch ablehnen kann, ohne davon einen Nachteil zu haben, würde man hingegen eher von Bitte sprechen. ${ }^{5}$ Während Befehle naturgemäß keine abtönenden Mittel erforderlich machen - ein vom Kommandanten an die Truppe gerichtetes Würden Sie bitte rechtsum kehrt machen? oder Könnten die bitte so nett sein und die Hände heben? als Aufforderung eines bewaffneten Räubers klänge absurd oder ironisch - ist dies bei Bitten anders: Hier kommen regelmäßig konventionalisierte Mittel zum Einsatz. Dies ist auch der Grund, weswegen sich dieser Sprechakt so gut für sprachvergleichende Untersuchungen (wie z. B. Màrquez Reiter 2000) oder auch für Spracherwerbsstudien (wie z. B. Achiba 2003) eignet.

\section{Die Erhebung}

Auch die vorliegende Untersuchung befasst sich daher mit Bitten; konkret geht es um die Art und Weise, wie sie in zwei Sprachen und drei Ländern, nämlich im deutschsprachigen Teil der Schweiz, in Deutschland und in Serbien geäußert werden. Methodisch stehen für eine Untersuchung dieser Art unterschiedliche Ansätze zur Verfügung. Von der Möglichkeit, literarische Texte im Hinblick auf den sprachlichen Ausdruck von Bitten auszuwerten, hat beispielsweise Milosavljević (2007: 37-79) Gebrauch gemacht. Eine andere Möglichkeit bieten Materialsammlungen, die mittels teilnehmender Beobachtung mit oder ohne verdeckte Aufnahmen erstellt werden (so z. B. bei Schlund 2009; in geringerem Umfang und ohne konkrete Quellenangaben ${ }^{6}$ auch bei Milosavljević 2007). Besonders bei der Untersuchung der sprachlichen Fähigkeiten und Strategien Fremdsprachenlernender sind ferner auch Rollenspiele beliebt (cf. z. B. Warga 2004; Saldago 2011); und last but not least bietet sich auch die Arbeit mit Fragebögen verschiedener Art an (cf. z. B. Olgiermann 2009, Economidou-Kogetsidis 2010 u. a. m.). ${ }^{7}$

Da verdeckte teilnehmende Beobachtung die besten, da "echtesten" Ergebnisse zu erbringen verspricht, sollte sie ursprünglich auch bei der hier vorgelegen Untersuchung Anwendung finden. Die Verfasserin führte daher eine kleine Pilotstudie in Berlin durch, indem sie sich einmal im U-Bahnhof Rudow, einmal in der Fußgängerzone in der Wilmersdorfer Straße je-

\footnotetext{
${ }^{5}$ Die im Lexikon gespiegelte Konzeptionalisierung von Sprechakten wie auch die dort gespeicherten Höflichkeitskonzepte können als wertvolle Hinweise auf die Wahrnehmung durch die jeweilige Sprach- und Kulturgruppe angesehen werden (cf. z. B. Pizziconi 2007).

${ }^{6}$ Die Angaben beschränken sich auf allgemeine Hinweise wie: "jüngere Frau zu einem unbekannten Mann" oder "Stewardess zu Passagier" (Milosavljević 2007: 50, Übersetzung E. H.)

$7 \mathrm{Zu}$ einer Abwägung schriftlicher Befragungen vs. Beobachtungen natürlicher sprachlicher Äußerungen cf. Beebe/Clark Cummings (1996/2006).
} 
weils neben eine Schlange von Menschen stellte, die vor einer Bäckerei anstanden. Dabei versuchte sie den Eindruck zu erwecken, in die Auslage vertieft und noch nicht recht sicher zu sein, was sie kaufen wollte. In einem Fall war es so möglich, sieben Personen beim Einkauf zu beobachten; im anderen waren es nur vier, bevor die Verkäuferin sich an die scheinbar unentschlossene Kundin wandte und fragte, ob sie helfen könne. In beiden Fällen äußerten sämtliche beobachteten Personen ihren Wunsch mit der identischen Formulierung Ich hätte gern (gefolgt von fünf Schrippen, eine Streuselschnecke, zwei Schusterjungs etc. - was immer die betreffende Person zu kaufen wünschte). Das scheint auf den ersten Blick ein wirklich bemerkenswertes und sehr vielversprechendes Ergebnis zu sein. Aber genau die Tatsache, dass alle jeweils die identische Formulierung wählten, gibt auch Anlass dazu, es noch einmal zu überdenken. Da alle Beobachteten jeweils gehört hatten, was die Person geäußert hatte, die vor ihnen an der Reihe war, ist nicht nur nicht auszuschließen, sondern sogar naheliegend, dass hier Priming-Effekte vorliegen könnten, wie sie ja für alle Ebenen sprachlicher Äußerung von der Wortwahl bis zur Wahl der syntaktischen Konstruktion gut belegt sind (cf. z. B. Gries 2013). Das entwertet solche Ergebnisse natürlich, denn vielleicht hätten die Beobachteten ja ohne das Priming der vorangehenden Äußerung etwas ganz anderes gesagt. Alternativ müsste man daher versuchen, den jeweils ersten Kunden zu belauschen, der nach einer Flaute an den Stand herantritt, um etwas zu kaufen - eine sehr aufwendige und daher wenig praktikable Methode.

Auch Fragebögen haben als Erhebungsmethode naturgemäß Nachteile, ganz besonders dann, wenn sie mit einer persönlichen Begegnung zwischen Fragenden und Befragten verbunden sind. Man kann das Problem des Beobachterparadoxons aber ein wenig entschärfen, indem man die Befragung in anonymisierter Form durchführt. Daher wurde eine zweite Pilotstudie mit anonymisierten Fragebogen durchgeführt und als Methode der vorliegenden Untersuchung schließlich die Form der Befragung per Internet gewählt. Dafür wurden insgesamt sechs alltägliche Situationen vorgestellt, in denen die Probanden jeweils eine Bitte äußern sollte. Die hier ausgewählten Situationen, um die es im Folgenden gehen soll, betrafen:

- die Frage nach dem Weg zum Bahnhof in einer fremden Stadt;

- die an den mit am Familientisch sitzenden Bruder gerichtete Bitte um Salz;

- den Kauf einer Brezel (in der deutschen Version) bzw. einer kifla (etwa: 'Kipfel', 'Hörnchen' in der serbischen Version).

Die zugehörigen Teile des Original-Fragebogens in beiden Sprachen finden sich im Anhang.

Insgesamt haben 336 Personen den Fragebogen ausgefüllt. ${ }^{8}$ Im Einzelnen waren dies:

- 202 Personen aus Deutschland (davon 39 m, 163 w)

- 68 Personen aus der Schweiz ${ }^{9}$ (davon 27 m, 41 w)

- 66 Personen aus Serbien (davon 11 m, 40 w, 15 ohne Angabe des Geschlechts)

Die Altersverteilung in den drei Gruppen war fast identisch: Mit zwei Ausnahmen, die sich unter den Deutschen fanden, waren alle Teilnehmenden, die Angaben zu ihrem Alter gemacht

\footnotetext{
${ }^{8} \mathrm{Zu}$ den nachfolgenden Statistiken ist anzumerken, dass nicht alle Teilnehmenden alle Fragen beantwortet haben, so dass sich nicht immer dieselbe Gesamtzahl ergibt.

${ }^{9}$ Wenn hier und im Folgenden von der Schweiz die Rede ist, ist stets die Deutschschweiz gemeint; in anderssprachigen Landesteilen wurde die Befragung nicht durchgeführt.
} 
haben, unter 35 Jahre alt, wobei die überwiegende Teil wiederum zur jüngeren Altersgruppe (18-25 Jahre) zählte. Das ist insofern nicht verwunderlich, als die Fragebogenadresse über Universitäten verbreitet wurde; die Mehrzahl der Antwortenden waren somit Studierende. Diese Verteilung bedeutet einerseits, dass das hier erhobene sprachliche Verhalten nicht notwendig das der gesamten jeweiligen Sprachgruppe repräsentiert, sondern nur das einer jüngeren, gebildeten Sprechergruppe. Andererseits führt sie aber auch dazu, dass die erhobenen Daten sehr gut untereinander vergleichbar sind, da die Sprechergruppen in allen drei Ländern kompatibel sind.

\subsection{Szene (1): Auf der Suche nach dem Bahnhof}

Die erste Situation, die im Fragebogen beschrieben war, lautete: "Sie wollen in einer fremden Stadt nach dem Weg zum Bahnhof fragen. Sie sprechen einen älteren Herrn an, der Ihnen zufällig begegnet, und sagen: ....". ${ }^{10}$ Mit der Vorgabe, dass es sich bei der angesprochenen fremden Person um einen älteren Herrn handeln sollte, sollte eine möglichst große Distanz hergestellt werden.

Einige deutschsprachige Teilnehmende an der Untersuchung haben den Hinweis auf die fremde Stadt so gedeutet, dass man dort sicherlich auch kein Deutsch verstehen würde, und ihre Frage daher auf Englisch formuliert. So lautete etwa die Antwort einer Schweizerin: "Excuse me. Can you tell me the way to the trainstation? (Und dann in jeder anderen mir geläufigen Sprache, bis in seinen Augen Verständnis aufblitzt)". Umgekehrt haben Befragte aus der Schweiz ihre Antwort aber auch häufig in ihrem jeweiligen Dialekt formuliert und damit gezeigt, dass sie eine fremde Stadt im eigenen Land und nicht im Ausland (auch nicht im deutschsprachigen Ausland, wo die Schweizer Dialekte mehrheitlich nicht verstanden werden) vor Augen hatten. Beide Strategien illustrieren aber, dass die Befragten sich offenbar ernsthaft in die beschriebene Situation hineinversetzt haben und dass die Antworten daher vermutlich auch dem entsprechen, was sie jeweils in der Realität sagen würden.

Bei der Auswertung der Ergebnisse zeigt sich zunächst eine sehr weitgehende Einheitlichkeit in der Art, wie die Kontaktaufnahme erfolgt: Man äußert in allen drei Gruppen mit deutlicher Mehrheit als erstes eine Entschuldigung. Bei den Deutschen ${ }^{11}$ war dies in $87 \%$ aller Antworten der Fall, wobei die Frauen mit 89\% noch etwas häufiger zu dieser Form der Gesprächseröffnung griffen als die Männer (76\%). Innerhalb der Sprechergruppe, die als erstes eine Entschuldigung äußerte, wurden die folgenden sprachlichen Formen gewählt:

$\begin{array}{ll}\text { Entschuldigung } & 48 \% \\ \text { entschuldigen Sie bitte } & 23 \% \\ \text { entschuldigen Sie } & 29 \%\end{array}$

Anders als bei den deutschen Befragten, wo nur zwei Personen bei ihrer Frage nach dem Weg zum Bahnhof einen Dialekt (Bairisch bzw. Schwäbisch) gebrauchten, tat dies etwas mehr als

\footnotetext{
10 Auf Serbisch: «Nalazite se u nepoznatom gradu i tražite železničku stanicu/kolodvor. Kad ugledate jednog starijeg gospodina, priđete mu i kažete: ...».

${ }^{11}$ Die deutschen Auskunftspersonen kamen mehrheitlich aus dem Bundesland Nordrhein-Westfalen. Es ist daher keineswegs auszuschließen, dass eine andere regionale Verteilung zu einer Veränderung der Ergebnisse führen könnte. In den vorliegenden Daten waren aber nicht genug Teilnehmende aus anderen Regionen enthalten, um eine entsprechende Auswertung zu rechtfertigen.
}

ISSN 1615-3014 
die Hälfte der Schweizer Befragten. Unterschiede zwischen den Geschlechtern ließen sich hier nicht beobachten. Vom Prinzip her ist das Gesamtbild, das sich in der Schweiz ergibt, indessen dem aus Deutschland sehr ähnlich: 88\% beginnen mit einer Entschuldigung, wobei die Verteilung auf Männer und Frauen hier keinen Unterschied zeigt. Allerdings fällt auf, dass bitte in der Schweiz deutlich seltener verwendet wurde: 29\% der Deutschen, aber nur 2\% der Schweizer fügten ein bitte an ihre Entschuldigungsformel an. Im Einzelnen ergab sich folgende Verteilung der gewählten Formen:12

$\begin{array}{lr}\text { Entschuldigung } & 64 \% \\ \text { excusez } & 24 \% \\ \text { entschuldigen Sie } & 10 \% \\ \text { entschuldigen Sie bitte } & 2 \%\end{array}$

Auch bei den serbischen Befragten ${ }^{14}$ hat mit $73 \%$ die überwältigende Mehrheit als erstes eine Entschuldigung geäußert. Weitere 18\% äußerten die Entschuldigung im Anschluss an einen vorangehenden Gruß, ein Phänomen, das sich auch bei drei Schweizern sowie bei einem Deutschen zeigte. Ferner hielten $6 \%$ einen Gruß als Kontaktaufnahmesignal ohne nachfolgende Entschuldigung für ausreichend (Schweizer: 4\%; Deutsche: 3\%).

Berücksichtigt man wie im Vorigen nur diejenigen Antworten, die mit einer Entschuldigung begannen, so ergibt sich die folgende Verteilung:

$\begin{array}{lr}\text { izvinite ['entschuldigen Sie'] } & 81 \% \\ \text { oprostite ['verzeihen Sie'] } & 6 \% \\ \text { izvinite, molim Vas ['entschuldigen Sie bitte'] } & 6 \% \\ \text { izvini ['entschuldige'] } & 2 \% \\ \text { ja se izvinjavam ['ich entschuldige mich'] } & 2 \%\end{array}$

Vergleicht man alle drei Gruppen, so zeigt sich ein leichtes Ungleichgewicht in der Wahl der Entschuldigung gegenüber dem Gruß. Insgesamt kann man aber dennoch ganz eindeutig sagen, dass die Entschuldigung der überwiegende erste Sprechakt ist, erst mit weitem Abstand gefolgt von einem Gruß, an den sich nicht wiederum eine Entschuldigung anschließt. Als einzig bemerkenswerten Unterschied zwischen den Sprachgruppen kann man festhalten, dass die Beliebtheit des Grußes als Mittel der Anrede eines Fremden sich unterscheidet: Am wenigsten machen Deutsche davon Gebrauch und am ehesten serbisch Sprechende, die den Gruß dabei teilweise auch als ausreichend empfinden, so dass keine Entschuldigung mehr angefügt wird. Allerdings muss dieser Unterschied angesichts der unterschiedlichen Samplegrößen vorsichtig bewertet werden.

\footnotetext{
12 Mit Ausnahme von excusez, das einzeln aufgeführt wird, wurden in regionalen Varianten geäußerte Formen wie Tschudiguung, Tschoudigong u. Ä. zur besseren Übersichtlichkeit unter der standarddeutschen Variante (hier: Entschuldigung) zusammengefasst.

${ }^{13}$ Die französische, von den Dialektsprechern auch oft in anderer und recht fantasievoller Weise geschriebene Entschuldigungsformel (es fanden sich: excuse, excüse, exgüsé, äxgüsi, exgüsi und egsgüse) findet sich in der Schweiz häufig und wird als Ersatz für entschuldigen Sie bzw. seine dialektalen Entsprechungen verwendet.

${ }^{14}$ Da ein großer Anteil der Befragten keine Angaben zum Geschlecht gemacht hat, wird hier auf eine entsprechende Auswertung verzichtet.

15 Die Verwendung der familiären 2. Person Singular kam zweimal vor und war in beiden Fällen gefolgt von der Anrede čiča (etwa: 'Onkel'). Zur Funktion dieser Anrede cf. Hentschel (2012).
} 


\begin{tabular}{|l|c|c|c|c|}
\hline & $\begin{array}{c}\text { Entschuldigung } \\
\text { zuerst }\end{array}$ & $\begin{array}{c}\text { erst Gruß, dann Ent- } \\
\text { schuldigung }\end{array}$ & $\begin{array}{c}\text { nur } \\
\text { Gruß }\end{array}$ & $\begin{array}{c}\text { Entschuldigung und } \\
\text { 'bitte' }\end{array}$ \\
\hline Deutschland & $87 \%$ & $1 \%$ & $3 \%$ & $23 \%$ \\
\hline Schweiz & $88 \%$ & $5 \%$ & $4 \%$ & $2 \%$ \\
\hline Serbien & $73 \%$ & $14 \%$ & $6 \%$ & $6 \%$ \\
\hline
\end{tabular}

Tabelle 1: Gruß und Entschuldigung

Nach der Entschuldigung und/oder dem Gruß, aber auch in den wenigen Fällen, in denen beides entfiel, musste nun natürlich das Anliegen selbst genannt werden. ${ }^{16}$ Hier ergab sich abermals eine große Einheitlichkeit in der Wahl der Mittel. Von den Deutschen wählten 99\% einen Interrogativsatz. Dabei setzten 79\% von ihnen das Modalverb können und eine hörerorientierte Formulierung ein, verwendeten also können/könnten Sie, wobei sich eine leichte Abweichung in Abhängigkeit vom Geschlecht ergab: 83\% der Frauen und 70\% der Männer verwendeten diese Form. Seltenere, ebenfalls hörerorientierte Frageformen waren wissen Sie...? (gewählt von insgesamt 7\%), kennen Sie...? (2\%) oder würden Sie ...? $(<1 \%)$. Daneben gab es einige wenige sprecherorientierte Fragen mit den Modalverben dürfen und können, also darf/dürfte/kann ich (Sie etwas fragen/Sie kurz stören)? (insgesamt 3\%). Ebenfalls sprecherorientiert, aber ohne Modalverb formulierten 5\% mit Wie komme ich zum Bahnhof? Die restlichen Fragen waren neutrale Fragen nach dem gesuchten Sachwissen wie Wo geht es hier zum Bahnhof? (3\%), die auch Abtönungspartikeln enthalten konnten (Wo ist denn hier der Bahnhof?). Von der Minderheit, die keinen Interrogativsatz wählte, äußerte immerhin noch 1\% eine Metafrage: Ich hätte eine Frage. Daneben fand sich bei zwei Befragten ein schlichtes Ich suche den Bahnhof. Einen Imperativ - also etwa eine Formulierung wie Bitte sagen Sie mir, wo es hier zum Bahnhof geht - wählte keine einzige Person.

Bei den Formen des Modalverbs können überwog der Indikativ mit knapp 53\% leicht gegenüber dem Konjunktiv. Dabei ist das Verhältnis bei Männern und Frauen umgekehrt, der Unterschied kann aber wegen der unterschiedlichen Samplegröße bei den beiden Geschlechtern nicht als signifikant angesehen werden:

\begin{tabular}{|l|c|c|}
\hline & Können Sie? & Könnten Sie? \\
\hline Deutschland insgesamt & $53 \%$ & $47 \%$ \\
\hline Frauen & $52 \%$ & $48 \%$ \\
\hline Männer & $46 \%$ & $54 \%$ \\
\hline
\end{tabular}

Tabelle 2: Konjunktivgebrauch in Deutschland

Auch Schweizerinnen und Schweizer griffen in 97\% der Fälle zum Mittel der Frage. Insgesamt 68\% der Befragten, 56\% der Männer und 76\% der Frauen, fuhren mit Könnten Sie oder

\footnotetext{
16 Bei einigen der Befragten hört der Eintrag allerdings nach der Entschuldigung auf; anscheinend nahmen sie an, dass die Fortsetzung mit der Frage nach dem Weg sich dann von selbst verstehe und nicht mehr ausformuliert werden müsste. Andererseits gab es in allen drei Gruppen jeweils auch einige wenige Personen, die an die Entschuldigung noch eine Erklärung des Typs ich bin fremd hier anfügten, bevor sie die Frage nach dem Weg formulierten.
} 
Können Sie (bzw. einer dialektalen Entsprechung wie chönted Sie oder chöit dr) fort. Dabei überwog auch hier der Indikativ mit 53\% aller Formen den Konjunktivgebrauch leicht, und abermals ist das Verhältnis bei Männern und Frauen umgekehrt, ohne dass man angesichts der geringen Zahl der Befragten hieraus weiterreichende Schlüsse ziehen könnte:

\begin{tabular}{|l|c|c|}
\hline & Können Sie? & Könnten Sie? \\
\hline Schweiz insgesamt & $53 \%$ & $47 \%$ \\
\hline Frauen & $42 \%$ & $58 \%$ \\
\hline Männer & $57 \%$ & $43 \%$ \\
\hline
\end{tabular}

Tabelle 3: Konjunktivgebrauch in der Schweiz

Hörerorientiert waren hier $89 \%$ der Fragen, während 13\% sprecherorientiert waren; 3\% der Befragten wählten die im Hinblick auf Sprecher-/Hörerorientierung neutrale Frage Wo geht's hier zum Bahnhof? Die nicht als Frage formulierte sprecherorientierten Äußerung Ich suche den Bahnhof schließlich wurde von fünf Personen gewählt (7\%). Ein Imperativ war auch hier nicht zu beobachten.

Die serbischsprachigen Befragten verwendeten ausnahmslos Interrogativsätze, um ihr Anliegen zu formulieren; Assertionssätze des Typs 'ich suche den Bahnhof' fanden sich hier nicht. Abermals wurde mehrheitlich das Modalverb 'können' (moći) gebraucht, das 72\% aller Befragten in ihrer Äußerung verwendeten. Das Serbische kennt zwar keinen Konjunktiv, aber einen Konditional (oder, je nach Terminologie, potencial), der im gegebenen Kontext dieselben Funktionen erfüllt. Allerdings wurde dieser Modus deutlich seltener verwendet als der Konjunktiv im Deutschen:

možete li? [Indikativ]: $\quad 81 \%$

da li biste mogli? [Konditional]: $19 \%$

Ein möglicher Grund, warum der Konditional seltener gewählt wird, könnte hier jedoch auch formaler Art sein. Die Formenbildung erfolgt anders als im Deutschen analytisch und ist daher aufwendiger als der Indikativ (cf. mogli biste vs. možete).

Auch die sprechorientierten Äußerungen waren hier seltener als bei den beiden deutschsprachigen Gruppen: nur 6\% wählten die Formulierung Mogu li da Vas pitam nešto? ('Kann ich Sie etwas fragen?').

Insgesamt ergeben sich beim Vergleich der gewählten Modi des Verbs 'können' die folgenden Werte:

\begin{tabular}{|l|c|c|c|}
\hline & 'können' insgesamt & davon Indikativ & davon Konjunktiv/Konditional \\
\hline Deutschland & $79 \%$ & $53 \%$ & $47 \%$ \\
\hline Schweiz & $68 \%$ & $53 \%$ & $47 \%$ \\
\hline Serbien & $72 \%$ & $81 \%$ & $19 \%$ \\
\hline
\end{tabular}

Tabelle 4: 'können' im Vergleich

Die Fragen wurden in vielen Fällen zusätzlich mit weiteren Elementen wie vielleicht, wohl, zufällig, in der Schweiz auch äch/ächt ('wohl') bzw. im Serbischen možda als downtonern 
versehen. Da all diese Wörter dem Ausdruck der Möglichkeit dienen, wurden die Werte für vielleicht, wohl etc. hier zusammengefasst: ${ }^{17}$

Deutschland: $\quad 21 \%$

Schweiz: $\quad 16 \%$

Serbien: $\quad 5 \%$

Es zeigt sich, wie man sieht, eine absteigende Häufigkeit des Einsatzes solcher zusätzlichen Mittel. Dasselbe Phänomen spiegelt sich noch deutlicher - und hier nun auch signifikant - im Gebrauch eines bitte bzw. molim, das in die Frage zusätzlich eingefügt wurde:

Deutschland: $\quad 62 \%$

Schweiz: $\quad 7 \%$

Serbien: $\quad 3 \%$

Den Unterschied zwischen Deutschland und Serbien könnte man möglicherweise strukturell erklären. Molim ist sehr viel erkennbarer als bitte eine Verbform in der 1. Person Singular, und sie wird daher auch häufig mit einem Personalpronomen als Objekt verknüpft: molim te/vas 'ich bitte dich/euch'. Tatsächlich handelt es sich bei den wenigen Vorkommen von molim in der vorliegenden Untersuchung um die mit dem Objekt vervollständigte Form molim Vas, also um 'ich bitte Sie'. Das ist natürlich als vollständiger Satz, der parenthetisch in den Interrogativsatz eingeschoben wird, syntaktisch gesehen deutlich aufwendiger als ein in den Satz eingefügtes bitte. Tatsächlich mögen solche Faktoren durchaus eine Rolle spielen. Aber zumindest bei den Befragten aus Deutschland und der Schweiz muss es andere Gründe für die unterschiedliche Gebrauchshäufigkeit geben, denn hier ist die syntaktische Umgebung identisch - die Verwendung mit $62 \%$ vs. $7 \%$ jedoch äußerst unterschiedlich. Nun wäre theoretisch denkbar gewesen, dass das fehlende bitte durch den Gebrauch von Modalwörtern wie vielleicht ausgeglichen wird. Wie sich zeigt, ist hier aber ebenfalls eine höhere Frequenz in Deutschland als in der Schweiz zu verzeichnen. Was immer der Grund dafür sein mag: Man kann konstatieren, dass der Gebrauch von abschwächenden Elementen aller Art offenbar in der Schweiz deutlich weniger verbreitet ist als in Deutschland.

Damit lässt sich zusammenfassend feststellen: Die grundlegenden Mechanismen, die zur Anwendung kommen, wenn man einen unbekannten älteren Herrn nach dem Weg zum Bahnhof fragen möchte, sind in allen drei Sprachgemeinschaften eindeutig identisch: Die überwältigende Mehrheit hat jeweils das Muster gewählt, sich zunächst zu entschuldigen und sodann mit einer Frage fortzufahren, die das Modalverb 'können' enthält. Unterschiede ergaben sich dennoch im Detail, so etwa beim Grüßen und insbesondere beim Gebrauch von bitte bzw. molim.

\subsection{Szene (2): Am Frühstückstisch}

Gänzlich andere soziale Bedingungen als bei der Frage nach dem Weg zum Bahnhof waren in einer Szene kodiert, die folgendermaßen beschrieben wurde: "Sie sitzen mit Ihrer Familie beim Essen und brauchen das Salz, das am anderen Ende des Tisches steht. Sie bitten Ihren

\footnotetext{
${ }^{17}$ Im Einzelnen fanden sich unter den vollständigen Antworten aus Deutschland 36 vielleicht, 5 zufällig und 1 wohl; bei denen aus der Schweiz 10 äch/ächt und 1 zufällig; bei denen aus Serbien 3 možda.
} 
jüngeren Bruder Daniel darum, indem Sie sagen:... ". ${ }^{18}$ Die soziale Distanz ist damit sehr gering, und die relative Intimität der Situation wird dann auch sogleich dadurch deutlich, dass jeweils fast die Hälfte der Befragten den in der Frage vorgegebenen Namen als Auftakt ihrer Äußerung verwendet hat, wobei in der Schweiz in einem Drittel der Fälle auch die familiäre Kurzform Dani eingesetzt wurde. Von den serbischen Befragten verwendeten $73 \%$ den Vornamen des Bruders, der hier Saša hieß, als Auftakt zur Kontaktaufnahme. Dabei wurde in zwei Fällen ein eher ungewöhnlicher Vokativ Sašo, in fünf weiteren die familiäre Kurzform Sale eingesetzt:

\begin{tabular}{|l|l|l|r|}
\hline Anrede mit Namen & W & m & gesamt \\
\hline Deutschland & $53 \%$ & $42 \%$ & $46 \%$ \\
\hline Schweiz & $44 \%$ & $16 \%$ & $43 \%$ \\
\hline Serbien & \multicolumn{3}{|l}{$73 \%$} \\
\hline
\end{tabular}

Tabelle 5: Anrede mit Namen

Eine Entschuldigung, die bei der Frage nach dem Weg zum Bahnhof Standard war, wurde hingegen von den deutschsprachigen Befragten in keinem einzigen Fall geäußert, und sie fand sich auch im Serbisch nur einmal (Saša, izvini sto te prekidam u jelu, da li mozeš da mi dodaš so? ('Saša, entschuldige, dass ich dich beim Essen unterbreche, kannst du mir das Salz reichen?'). Gleich blieb hingegen die Bevorzugung der Frageform gegenüber einem möglichen Imperativ, die wiederum mit einem Modalverb und/oder dem Gebrauch des Konjunktivs verknüpft sein kann. Mehrheitlich gewählt wurden dabei die folgenden hörerorientierten Formulierungen: 19

\begin{tabular}{|c|c|c|c|}
\hline Frage mit 'können' im Indikativ: & $\mathrm{w}$ & $\mathrm{m}$ & gesamt \\
\hline Deutschland & $44 \%$ & $47 \%$ & $52 \%$ \\
\hline Schweiz & $25 \%$ & $17 \%$ & $21 \%$ \\
\hline Serbien & & & $19 \%$ \\
\hline
\end{tabular}

Fragen mit 'können' im Konjunktiv/Konditional:

\begin{tabular}{|l|r|r|r|}
\hline Deutschland & $5 \%$ & $3 \%$ & $5 \%$ \\
\hline Schweiz & $20 \%$ & $29 \%$ & $22 \%$ \\
\hline Serbien & & & $5 \%$ \\
\hline
\end{tabular}

\footnotetext{
${ }^{18}$ Serbisch: "Sedite s porodicom za ručkom i zatreba Vam so, koja se nalazi na drugom kraja stola pored Vašeg mlađeg brata Saše. Okrenete mu se i kažete: ..."

${ }^{19}$ Daneben traten auch die sprecherorientierten Formen Kann/könnte ich haben?, Krieg/bekomme ich? sowie die hörerorientierten Fragen Gibst du mir? und Hast du mir? (Hesch mer?) auf.
} 
Verwendung des Imperativs:

\begin{tabular}{|l|r|r|r|}
\hline Deutschland & $30 \%$ & $37 \%$ & $31 \%$ \\
\hline Schweiz & $3 \%$ & $8 \%$ & $4 \%$ \\
\hline Serbien & & & $73 \%$ \\
\hline
\end{tabular}

Tabelle 6: Indikativ, Konjunktiv, Imperativ

Hier zeigt sich nun anders als im ersten Fall ein deutlicher Unterschied in der Häufigkeit des Konjunktivgebrauchs bei den beiden deutschsprachigen Gruppen, und auch die Verwendung des Imperativs weicht in der Frequenz stark voneinander ab. Mit dem Imperativ verbindet sich bei den deutschsprachigen Befragten zugleich ein jeweils ganz unterschiedlicher Gebrauch der Abtönungspartikel mal. In Deutschland setzen zwei Drittel sie ein, um entweder die Imperative (gib mir sowie seltener auch reich mir) abzutönen - Imperative ohne mal waren nicht zu beobachten - oder auch um die in Frageform gekleidete Aufforderung noch weiter abzutönen. Von den Schweizer Befragten fügten nur 4\% ein mal zu ihrer Äußerung hinzu, und hier findet sich auch ein Imperativ ohne mal (dafür allerdings mit bitte).

mal:

Deutschland $\quad 67 \%$

Schweiz $\quad 4 \%$

Sehr interessant sind auch die Veränderungen, die beim Gebrauch von bitte im Vergleich mit der vorigen Situation ergeben. Während die Frequenz dieses Wortes bei den Deutschen, wo $62 \%$ das Wort in ihre Frage nach dem Weg eingefügt hatten, mit nunmehr 65\% praktisch gleich bleibt, zeigt sich bei den Schweizer Befragten plötzlich ein starker Anstieg: von 7\% auf $55 \%$. Und auch im Serbischen steigt die Frequenz von molim von nur 3\% bei der Frage nach dem Weg zu Bahnhof auf nunmehr immerhin 19\%. Dabei machen Frauen in beiden deutschsprachigen Gruppen häufiger Gebrauch von bitte, als dies die Männer tun. Besonders deutlich zeigt sich dieser Unterschied in der Schweiz:

\begin{tabular}{|l|l|l|r|}
\hline bitte/molim & $\mathrm{W}$ & $\mathrm{m}$ & gesamt \\
\hline Deutschland & $71 \%$ & $42 \%$ & $65 \%$ \\
\hline Schweiz & $64 \%$ & $15 \%$ & $55 \%$ \\
\hline Serbien & \multicolumn{3}{|c}{$19 \%$} \\
\hline
\end{tabular}

Tabelle 7: bitte/molim

Die gegenüber der zuvor beschriebenen Situation deutlich erhöhte Häufigkeit von bitte ist ein recht unerwartetes Ergebnis, denn eine intimere Situation mit geringerer sozialer Distanz würde normalerweise nicht mehr, sondern weniger abmildernde Elemente erforderlich machen. Eine mögliche Erklärung wäre, dass das Wort in den verschiedenen Regionen unterschiedlich konnotiert ist - etwa, indem es in der Schweiz und auch in Serbien einem eher familiären Register zugeordnet oder auch als fordernder empfunden wird - und daher unterschiedlich gut zu der jeweiligen Situation passt. Im Serbischen könnte allerdings auch die Tatsche eine Rolle spielen, dass Imperative hier überwiegen, die als gesichtsbedrohende Äußerungsform dann möglicherweise eher durch den Zusatz von 'ich bitte' abgemildert werden. 
Während $\mathrm{mal}$ in der Schweiz nur selten verwendet wird, setzen dort 15\% der Befragten die nur hier gebräuchliche Partikel äch/ächt ('wohl') ein, und weitere 24\% markieren die Aufforderung mit schnell (bzw. schnäu), das in Deutschland nur eine Person verwendet hat. Allem Anschein nach kommen hier zwar vom Prinzip her sehr ähnliche, in der Wortwahl dann jedoch deutlich unterschiedliche Strategien zur Anwendung. Insbesondere schnell/schnäu und mal beruhen ja auf denselben semantischen Mechanismen: Die Handlung wird mit mal als 'einmalig' und damit indirekt zugleich als 'nur kurz andauernd' markiert, um damit das Ausmaß der Forderung zu reduzieren und so die Bitte "kleiner" zu machen.

Wie bereits erwähnt war die häufigste Strategie, die von den serbischen Befragten gewählt wurde, anders als in den anderen beiden Gruppen der Imperativ. Fast 3/4 der Befragten äußerten entweder daj mi so ('gib mir das Salz') oder dodaj mi so ('reiche mir das Salz') geäußert. Die Verben dati und dodati sind beide perfektiv, und die Aufforderung war mehrheitlich von einer Anrede mit dem Namen eingeleitet. Offenbar wurden diese beiden Elemente, also die Verwendung eines perfektiven Verbs und die persönliche Anrede, von vielen als ausreichend angesehen, um die Bitte im gegebenen Kontext akzeptabel zu machen. Die Bandbreite der unterschiedlicher Möglichkeiten, die im Serbischen verwendet wurden, war ansonsten aber insgesamt recht groß. Sie reichen von einem sehr kurz angebundenen daj vamo (etwa: 'gib mal her') oder einem ebenfalls knappen, von keinen weiteren Elementen begleiteten dodaj mi so (etwa: 'Gib mir mal das Salz') über Fragen des Typs 'Kannst du mir das Salz geben?' bis hin zu Formulierungen wie Saša, da li bi bio ljubazan da mi dodaš so? Hvala ('Saša, wärst du so nett, mir das Salz zu geben? Danke') und dem bereits erwähnten Saša, izvini sto te prekidam u jelu, da li mozes da mi dodas so? ${ }^{20}$ ('Saša, entschuldige, dass ich dich beim Essen unterbreche, kannst du mir das Salz reichen?').

\subsection{Szene (3): Beim Bäcker}

Im zu Beginn dieses Artikels angeführten Zitat äußerte der Autor die Vermutung, dass man seinen Kaffee auf Deutsch je nach nationaler Zugehörigkeit mit oder ohne Konjunktiv bestellt. Abgesehen von der Nationalität dürften bei der Kaffeebestellung jedoch auch noch andere Faktoren bei der Wahl der sprachlichen Form eine Rolle spielen, so etwa die Tageszeit und die räumliche Umgebung. Nachdem man in einem gehobenen Restaurant in eleganter Kleidung und bei Kerzenschein ein mehrgängiges Diner eingenommen hat, bestellt man den Kaffee vermutlich nicht mit genau denselben Worten wie am frühen Morgen am Bäckerstand oder bei MacDonalds an der Theke. Auch Kleidung und Auftreten der Bedienung können die Art der Bestellung sicherlich beeinflussen, so dass man beim Kellner im Frack vielleicht nicht dieselben Worte wählt wie bei der verschwitzten und abgehetzten Bedienung im überfüllten Biergarten. Zudem lässt sich vermuten, dass neben Kleidung und Auftreten auch das Sprachverhalten der Bedienung selbst einen Einfluss auf die Wortwahl der Bestellenden hat. ${ }^{21}$

\footnotetext{
${ }^{20}$ In allen Fällen wurde die Originalschreibweise beim Zitieren der Antwort beibehalten, so dass sich Schreibweisen mit diakritischen Zeichen neben solchen finden, in denen sie nicht enthalten sind.

${ }^{21}$ Ein im serbischen Sprachraum sehr bekanntes Beispiel, das diesen Effekt aufs schönste illustriert, findet sich in der berühmten Videofassung von Dušan Kovačevićs Drama Radovan Treći, wenn Tasko Načić als Kellner mit den Worten "Šta piju pičke?" (wörtlich: 'Was trinken die Fotzen?') nach den Wünschen dreier Damen am Tisch fragt - eine Formulierung, auf die ein 'Dürfte ich um einen Kaffee bitten?' als Antwort wohl ausgeschlossen werden kann.
} 
Um solche Faktoren weitestgehend auszuschließen und um in allen drei sprachlichen Umgebungen eine etwa gleiche Distanz zwischen den Beteiligten zu etablieren, wurde für die Befragung eine andere Situation gewählt. Statt um die Bestellung eines Kaffees ging es um den Kauf einer Brezel bzw. eines Hörnchens beim Bäcker, wo man zuvor in einer Schlange gewartet hat, um an die Reihe zu kommen. Damit ist die Situation relativ uniform und vor allem auch für alle betroffenen Kulturregionen vergleichbar. Die soziale Distanz dürfte in dieser Situation in der Mitte zwischen den beiden zuvor geschilderten Szenarien liegen. Die Vorgabe lautete auf Deutsch: "Sie wollen sich beim Bäcker eine Brezel kaufen. Als Sie an die Reihe kommen, sagen Sie...".22 Die nachfolgende Tabelle (8) zeigt eine zusammenfassende Statistik der Antworten in Deutschland und der Schweiz. Dabei wurden folgende sprachlichen Phänomene erfasst:

- Der Brezelbestellung geht ein Gruß (Guten Tag, Gruessech, ${ }^{23}$ Hallo o. Ä.) voraus.

- Das Wort bitte wird hinzugefügt.

- Es wird ein Konjunktiv verwendet. ${ }^{24}$

- Es erfolgt Kombination aus beiden vorgenannten Phänomenen: Das konjunktivische ich hätte gern wird von einem bitte begleitet.

- Es steht ein gern, jedoch kein ich hätte (also z. B.: en bräzel gärn 'eine Brezel gern')25

\begin{tabular}{|l|r|r|r|r|r|}
\hline & Gruß & bitte & Konjunktiv & $\begin{array}{c}\text { Kombination aus } \\
\text { Konjunktiv + bitte }\end{array}$ & $\begin{array}{c}\text { gern/gärn } \\
\text { (ohne Verb) }\end{array}$ \\
\hline D, w & $31 \%$ & $54 \%$ & $55 \%$ & $11 . \%$ & $0 \%$ \\
\hline D, m & $3 \%$ & $59 \%$ & $46 \%$ & $8 \%$ & $0 \%$ \\
\hline D, gesamt & $30 \%$ & $55 \%$ & $53 \%$ & $10 \%$ & $0 \%$ \\
\hline
\end{tabular}

\footnotetext{
22 Auf Serbisch: "Otišli ste kod pekara da kupite kiflu. Kad dođete na red, kažete..." (Übersetzung: 'Sie sind zum Bäcker gegangen, um ein Hörnchen zu kaufen. Als Sie an die Reihe kommen, sagen Sie...').

23 Berndeutscher Gruß; '(ich) grüße euch'.

${ }^{24}$ Mit zwei Ausnahmen - einmal ich möchte gerne, einmal ich nämmti gern ('ich nähme gern') - handelte es sich dabei stets um die Formel ich hätte gern(e).

${ }^{25}$ Die Formulierung Eine Brezel gern bzw. dialektale Varianten davon findet sich nur bei den Schweizer Teilnehmenden an der Umfrage. Sie ist auch dort nicht häufig; dass sie im sehr viel größeren deutschen Sample gar nicht auftritt, ist aber dennoch bemerkenswert. Offenbar ist sie nicht im gesamten Sprachgebiet gebräuchlich.
} 


\begin{tabular}{|l|r|r|r|r|r|}
\hline & Gruß & bitte & Konjunktiv & $\begin{array}{l}\text { Kombination aus } \\
\text { Konjunktiv + bitte }\end{array}$ & $\begin{array}{l}\text { gern/gärn } \\
\text { (ohne Verb) }\end{array}$ \\
\hline $\mathrm{CH}, \mathrm{w}$ & $65 \%$ & $5 \%$ & $83 \%$ & $0 \%$ & $10 \%$ \\
\hline $\mathrm{CH}, \mathrm{m}$ & $38 \%$ & $7 \%$ & $70 \%$ & $0 \%$ & $11 \%$ \\
\hline $\mathrm{CH}$, gesamt & $54 \%$ & $6 \%$ & $78 \%$ & $0 \%$ & $11 \%$ \\
\hline
\end{tabular}

Tabelle 8: Deutschsprachige Strategien beim Bäcker

Noch deutlicher als schon im Vorigen zeigt sich hier, dass die Sprache als solche nicht allein ausschlaggebend für die gewählte Formulierung ist. Man erkennt eine ganze Reihe von Unterschieden, nicht zuletzt auch solche zwischen den Geschlechtern. So scheinen etwa Frauen in beiden Sprachregionen häufiger zu grüßen als Männer, bevor sie ihren Wunsch äußern (sehr deutlich mit 31\% vs. 3\% in Deutschland, immer noch klar häufiger mit $65 \%$ vs. $38 \%$ in der Schweiz). Frauen aus der Schweiz grüßen jedoch immer noch doppelt so oft wie ihre Kolleginnen aus Deutschland, und auch die Männer sind hier eindeutig grußfreudiger. Allerdings sind die hier diskutierten Ergebnisse angesichts der sehr unterschiedlichen TeilnehmendenZahlen natürlich mit Vorsicht zu behandeln und müssen als vorläufig betrachtet werden.

Höchst bemerkenswert ist in dieser Situation nun abermals der äußerst unterschiedlich häufige Gebrauch von bitte bei den beiden Probandengruppen. Nur 6\% der Schweizer haben es verwendet, während $55 \%$ der Deutschen das Wort eingesetzt haben. Eine konjunktivische Äußerung wird hingegen von 78\% der Schweizerinnen und Schweizer, aber nur 53\% der Deutschen gewählt. Die sehr stark standardisierte Formel ich hätte gern, um die es sich dabei handelt, wird von den Befragten aus der Schweiz offenkundig als ausreichend angesehen, um das notwendige Maß an Höflichkeit zu wahren. Bitte wird hier nur eingesetzt, wenn kein Verb verwendet wird, also für die Formulierung Eine Brezel bitte und ihre mundartlichen Varianten.

Die in der Schweiz immer wieder als Standardvariante deutscher Sprecher kolportierte Formulierung ich kriege eine Brezel (cf. z. B. Reihl 2009, Klappentext) konnte demgegenüber in keinem einzigen Fall beobachtet werden. Dies widerspricht auch der Feststellung von Schlund (2009: 77), diese Konstruktion sei zusammen mit ich bekomme ebenso häufig anzutreffen wie ich hätte gerne. Allerdings wird diese Aussage auch durch ihr eigenes Korpus (cf. ibd.: 217252) nicht bestätigt, so dass unklar bleibt, auf welchen Daten sie beruht. Aber immerhin ist die Wendung in Schlunds Korpus - anders als in der vorliegenden Untersuchung - an einigen Stellen tatsächlich vorgekommen. Bemerkenswert ist dabei allerdings, dass sie sich niemals in initialer Verwendung findet, sondern stets erst nach einem vorgängigen Austausch, einmal sogar nach einem direkt im Turn zuvor geäußerten ich hätte gern (cf. ibd.: 222, 228, 230). Der Status einer Äußerung, die im Laufe des Gesprächs erfolgt, und einer mehr oder minder gesprächseröffnenden Bitte kann aber selbstverständlich nicht gleichgesetzt werden. Hier wären weitere Analysen nötig, um festzustellen, ob und wenn ja unter welchen Bedingungen Deutsche ein ich kriege als ersten Turn in einer Äußerung wirklich verwenden.

Die serbischen Probanden werden im Folgenden abermals nicht nach Geschlechtern unterschieden, da die entsprechenden Angaben zu häufig fehlen. Was hier neuerlich ins Auge fällt, ist wiederum die große Bandbreite der Formulierungen - es gibt offenbar anders als im Deutschen keine mehr oder weniger festen Formeln, die man einsetzen kann: 


\begin{tabular}{|l|c|}
\hline dobar dan ('guten Tag') & $39 \%$ \\
\hline molim/molim vas ('bitte') & $54 \%$ \\
\hline $\begin{array}{l}\text { jednu kiflu/za mene jedna kifla } \\
\text { ('ein Hörnchen'/ 'für mich ein Hörnchen') - ohne 'bitte' }\end{array}$ & $16 \%$ \\
\hline daćete mi ('Sie werden mir geben') & $16 \%$ \\
\hline ja ću ('ich will') & $7 \%$ \\
\hline dajte mi ('geben Sie mir') & $7 \%$ \\
\hline hteo bih/molio bih/ja bih (etwa: 'ich hätte gern') & $5 \%$ \\
\hline mogu li da dobijem? ('kann ich bekommen?') & $5 \%$ \\
\hline uzeću ('ich werde nehmen') & $2 \%$ \\
\hline
\end{tabular}

Tabelle 9: Gewählte Strategien auf Serbisch

Was den Gruß zu Beginn der Bestellung angeht, so sind die Zahlen hier etwas höher als bei den Deutschen (dort 30\%, hier 39\%). An die Häufigkeit des Grüßens, die sich bei den Schweizern gezeigt hat (54\%), reichen sie jedoch nicht heran; sie stehen so gesehen in der Mitte zwischen den beiden anderen Probandengruppen. Dafür ist der Gruß einheitlich; niemand hat etwas anderes als dobar dan verwendet. Dagegen variiert bei den deutschsprachigen Befragten die Form des Grußes sehr; es findet sich neben Guten Tag und Hallo auch regionale Varianten wie Grüssech, Grüß Gott oder Moin.

Die Konditionale hteo bih [etwa: 'ich würde wollen'], molio bih [etwa: 'ich würde bitten'], und ja bih [etwa: 'ich würde'] haben im gegebenen Kontext alle drei eindeutig dieselbe Funktion, die der deutsche Konjunktiv in der Formulierung ich hätte gern inne hat, und sind daher in der Tabelle auch zusammengefasst worden.

Eine nicht ohne Weiteres adäquat ins Deutsche übersetzbare Wendung besteht im Gebrauch des Futurs der 2. Person Plural (daćete mi, wörtlich 'Sie werden mir geben') in diesem Kontext. Übersetzt man diesen Aufforderungstyp wörtlich ins Deutsche oder ins Englische (Sie werden mir eine Brezel geben/you will give me a pretzel), so entsteht eine äußerst unhöfliche Äußerung, die eher nach einem Befehl klingt. ${ }^{26}$ Da die Formulierung im Serbischen jedoch als höflich wahrgenommen wird, ${ }^{27}$ muss angenommen werden, dass hier andere Mechanismen wirksam werden als in den Nachbarsprachen. Schlund (2009: 77) verwendet bei ihrer Diskussion dieses Phänomens den Ausdruck "modales Futur", der in diesem Kontext allerdings erklärungsbedürftig ist. Gewöhnlich ist damit der Gebrauch des Futurs zum Ausdruck einer Vermutung gemeint, wie er in der Mehrheit der Sprachen möglich ist (cf. Bybee/Dahl 1989). Diese modale Funktion des Tempus zeigt sich auch im Serbischen. Das erklärt aber

\footnotetext{
${ }^{26}$ Im Unterschied zu hierzu wäre im Französischen die entsprechende Äußerung Vous me donnerez un croissant zu Beginn des Verkaufsgesprächs weitgehend unverständlich; sie könnte aber im Verlauf eines umfassenderen Einkaufs erfolgen, wenn schon anderes gekauft wurde und nun ein weiterer Kaufwunsch im Sinne eines ,und dann noch ein Croissant' hinzugefügt wird.

${ }^{27}$ Interessanterweise wird die Verwendung des Futurs zum Ausdruck einer Bitte in informeller mündlicher Rede auch bei der Zusammenfassung der bestehenden Möglichkeiten bei Milosavljević (2007: 78) gar nicht erwähnt.
} 
noch nicht die hier diskutierte Verwendung, denn dass man auch im Deutschen Das wird der Postbote sein sagen kann, führt ja nicht dazu, dass eine Äußerung wie Sie werden mir eine Brezel geben als höflich wahrgenommen würde. Es liegt daher nahe anzunehmen, dass hier andere Mechanismen wirksam sind. Denkbar wäre, dass nicht nur die grammatikalisierte temporale Funktion von hteti 'wollen' zur Futurbildung wirksam ist, sondern dass die Bedeutung des Modalverbs hteti selbst noch mitverstanden wird. Die Futurbildung mit hteti und einem Vollverb impliziert ursprünglich, dass es sich beim ausgedrückten Geschehen um einen Wunsch der handelnden Person handelt. Dass diese Bedeutungskomponente auch gesichtsbewahrend wirksam gemacht werden kann, lässt sich gut am Beispiel des Englischen illustrieren, wo Aussagen über die Zukunft für die erste Person als Pflicht (I shall), für die zweite hingegen höflichkeitshalber als deren Wunsch (you will) grammatikalisiert wurden. In daćete $m i$ liegt nun allerdings eine kontrahierte Form vor, wie sie für den Ausdruck des Wunsches nicht verwendet wird. Es wäre indessen denkbar, dass die Bedeutung 'Wunsch' infolge des Nebeneinanders der beiden Funktionen ${ }^{28}$ mitschwingt. Wenn sozusagen unterstellt wird, dass die angesprochene Person die erbetene Handlung von sich aus gerne ausführen möchte, kann diese Formulierung als gesichtswahrend und höflich interpretiert werden.

Besonders interessante Unterschiede zeigen sich schließlich beim Vergleich der drei Gruppen im Hinblick auf den Gebrauch von bitte/molim: Während in dieser Situation mit 55\% der deutschen und 54\% der serbischen Befragten etwa gleich viele Personen es verwendet haben und damit offenbar dieselbe Höflichkeitsstrategie anwenden, findet sich bitte nur bei gerade einmal 6\% der Schweizer Befragten. Allem Anschein nach gelten hier wirklich andere Verwendungsbedingungen für das Wort, als dies bei den beiden anderen Gruppen der Fall ist. Dies ist um so bemerkenswerter, als sich hier innerhalb ein und derselben Sprache in zwei Ländern große Unterschiede zeigen, während der Vergleich zweier verschiedener Sprachen und Länder keine solchen Differenzen zu Tage fördert.

\section{Abschließende Bemerkungen}

Es böte sich nun an, einen Vergleich aller drei Situationen in Bezug auf die Frequenz des Gebrauchs bestimmter Strategien wie Einsatz des Konjunktivs/Konditionals oder Verwendung von Interrogativsätzen vs. Imperativsätzen bei den einzelnen Probandengruppen vorzunehmen. Dass dies jedoch höchst problematisch ist, weil die grundlegenden grammatischen Gegebenheiten im Deutschen und im Serbischen nicht vergleichbar sind, wurde im Vorigen am Beispiel des Imperativs bereits verdeutlicht. Insofern sind auch die nachfolgenden Vergleiche der Zahlen nur als Annäherung an die Phänomene gedacht; sie können nicht wirklich direkt aufeinander bezogen werden.

Was man insofern recht gut miteinander vergleichen kann, als es sich um übersetzungsäquivalente Formen handelt, ist der Gebrauch von bitte/molim. Beide Sprachen verfügen über den entsprechenden Ausdruck, der jeweils nicht syntaktisch in den Satz integriert wird und daher als Zusatz zu anderen Formen der Äußerung verwendet werden kann. Allerdings gibt es auch

\footnotetext{
${ }^{28}$ Auch das Futur kann in nicht-kontrahierter Form gebildet werden, und die Konstruktionen pevaću, hoću pevati/da pevam, ću pevati/da pevam ('ich werde singen') unterscheiden sich nur im Hinblick auf Stellungsmöglichkeiten ( $c u$ kann nur klitisch gebraucht werden und braucht daher beispielweise ein vorangestelltes $j a$ 'ich' oder sad 'jetzt') sowie auf Konnotationen (pevati vs. da pevam), nicht jedoch in ihrer Tempusbedeutung.
}

ISSN 1615-3014 
hier einen wichtigen Unterschied: Während die volle Form ich bitte dich bzw. ich bitte Sie im Deutschen markiert und zum Ausdruck einer Bitte nicht gebräuchlich ist, gilt dies nicht für die hochfrequenten serbischen Entsprechungen molim te und molim Vas. Dennoch zeigen die Zahlen, wie bereits erwähnt, hier wirklich interessante Unterschiede:

\begin{tabular}{|l|r|r|r|}
\hline bitte/molim & $\begin{array}{c}\text { (1) auf der Suche } \\
\text { nach dem Bahnhof }\end{array}$ & (2) bei Tisch & (3) in der Bäckerei \\
\hline Deutsche & $62 \%$ & $65 \%$ & $55 \%$ \\
\hline Schweizer & $7 \%$ & $55 \%$ & $6 \%$ \\
\hline Serben & $3 \%$ & $19 \%$ & $54 \%$ \\
\hline
\end{tabular}

Tabelle 10: bitte und molim in allen drei Situationen im Vergleich

Sowohl die serbischen als auch die Schweizer, nicht aber die deutschen Befragten machen beim Gebrauch von 'bitte' offensichtlich einen Unterschied zwischen der familiären Situation am häuslichen Tisch und der an einen Fremden gerichteten Frage nach dem Weg zum Bahnhof. Bei beiden Gruppen erhöht sich die Frequenz in Situation (2), bei der Schweizer Gruppe stark, bei der serbischen schwächer, was in letzteren Fall auf den gleichzeitigen Anstieg des Gebrauchs von Imperativen zurückzuführen sein könnte. Dass dies nicht die ganze Erklärung sein kann, zeigt die auffällige Häufigkeit des Gebrauchs von molim beim Gebäckkauf in Situation (3). Hier finden sich auch im Serbischen nur wenige Imperative; dennoch hat nun sogar mehr als die Hälfte der Befragten molim verwendet.

Eigentlich wäre die Annahme naheliegend gewesen, dass familiäre Vertrautheit grundsätzlich weniger abmildernde Markierungen einer Bitte nötig macht, als dies bei einer DistanzSituation der Fall ist. Verblüffenderweise zeigt sich aber, dass es in der Schweiz genau umgekehrt ist - Szene (2) ist hier die einzige, in der überhaupt ein häufiger Gebrauch von bitte auftritt - und dass sich auch in Deutschland die Anzahl der bitte-Verwendungen eher erhöht, als dass sie geringer wird. Im Serbischen hingegen werden allem Anschein nach Bitten um eine Handlung (das Gebäck/das Salz soll übergeben werden) von solchen um eine rein verbale Reaktion (der Weg zum Bahnhof soll beschrieben werden) unterschieden und daher unterschiedlich markiert. Dass Bitten um konkrete Handlungen mehr Abmilderungen verlangen als solche, die nur eine Sprechhandlung nötig machen, wäre nachvollziehbar. Die größere Distanz in der Bäckerei erhöht dann die Anzahl noch einmal gegenüber der Situation am Familientisch.

Selbstverständlich kann man bitte nicht gesondert betrachten, sondern muss dabei auch andere downtoner wie mal, časkom oder das in der Schweiz gebräuchliche äch/ächt mit berücksichtigen. Wenn man alle diese Elemente zusammenfasst, ergeben sich die folgenden Werte (ohne bitte/molim):

\begin{tabular}{|l|c|c|c|}
\hline & $\begin{array}{c}\text { (1) auf der Suche } \\
\text { nach dem Bahnhof }\end{array}$ & (2) bei Tisch & (3) in der Bäckerei \\
\hline Deutschland & $21 \%$ & $68 \%$ & $0 \%$ \\
\hline Schweiz & $16 \%$ & $39 \%$ & $0 \%$ \\
\hline Serbien & $5 \%$ & $5 \%$ & $0 \%$ \\
\hline
\end{tabular}

Tabelle 11: Downtoner in allen drei Situationen im Vergleich 
Offenkundig macht die Situation in der Bäckerei in allen drei Gruppen keine zusätzlichen Elemente erforderlich. Dies erscheint insofern nachvollziehbar, als es ja um eine Handlung geht, die von der angesprochenen Person berufsmäßig ausgeführt wird.

Aber abgesehen von dieser Parallele müssen auch diese Werte natürlich im Kontext der Gesamtäußerungen interpretiert werden. So werden etwa im Serbischen zwar bei der Frage nach dem Weg zum Bahnhof kaum downtoner benutzt; anderseits fällt aber die ungewöhnlich Länge der Äußerungen auf: Sie umfassten zwischen 8 und 31 Wörtern ${ }^{29}$ (im Durchschnitt: 14), was bei einer artikellosen pro-drop-Sprache beachtlich ist. Bei der Frage nach dem Salz umfasste die längste Äußerung 14 Wörter, die zweitlängste 11, und der Rest bewegte sich zwischen 3 und 9 Wörtern; beim Bäcker waren die vier längsten Äußerungen 8, 9 und 10 Wörter lang, wobei sie aber auch Grüße oder Zusatzbestellungen enthielten, ${ }^{30}$ und der Rest war 2-6 Wörter lang. Es ist offensichtlich, dass hier noch mit anderen Mitteln als mit Modalwörtern oder Abtönungspartikeln gearbeitet wird - übrigens auch bei den beiden deutschsprachigen Gruppen, die hier ebenfalls die längsten Äußerungen getätigt haben. Aber dass die Länge der serbischen die der Antworten beider deutschsprachigen Gruppen (maximal 21 Wörter, im Durchschnitt 10 Wörter pro Äußerung) ${ }^{31}$ deutlich übersteigt, kann nur als Hinweis darauf gewertet werden, dass hier eine relativ aufwendige Gesprächsarbeit geleistet wird.

Fazit: Die gewählten Strategien unterscheiden sich in der Tat, wenngleich vielleicht anders als erwartet und in Abhängigkeit von der jeweiligen Situation in verschiedener Art und Weise. Der Vergleich der sprachlichen Formen stößt dabei zugleich sehr schnell an sehr weitgehende Grenzen.

\section{Literaturverzeichnis}

Achiba, Machiko (2003): Learning to Request in a Second Language: A Study of Child Interlanguage Pragmatics. Clevedon etc.: Multilingual Matters.

Bakšić, Sabina (2012): Strategije učtivosti u turskom jeziku. Sarajevo: Filozofski fakultet u Sarajevu.

\footnotetext{
${ }^{29}$ Die 31 Wörter umfassende Äußerung lautete: Dobar dan, gospodine, da li mozete da mi pomognete? Nisam odavde i ne mogu da nadjem zeleznicku stanicu, pa ako biste bili radi da mi pomognete, bila bih Vam veoma zahvalna (etwa: 'Guten Tag, mein Herr, können Sie mir helfen? Ich bin nicht von hier und ich kann den Bahnhof nicht finden, wenn Sie also so nett wären, mir zu helfen, wäre ich Ihnen sehr dankbar'). An zweiter Stelle in der stand die 23 bzw. 25 Wörter umfassenden Äußerungen Izvinite, da li biste mogli da mi pomognete? Nisam odavde, a moram da stignem na voz. Da li mozete da me uputite kuda da idem? (etwa: 'Entschuldigen Sie, könnten Sie mir helfen? Ich bin nicht von hier, und ich muss einen Zug erreichen. Können Sie mir zeigen, wohin ich gehen muss?') und Izvinite, da li mogu nešto da Vas pitam, gde se nalazi železnička stanica? Nisam odavde, pa mi je potrebna mala pomoć, hvala Vam! (etwa: 'Entschuldigen Sie, kann ich Sie etwas fragen, wo befindet sich der Bahnhof? Ich bin nicht von hier, da brauche ich etwas Hilfe, danke schön!').

30 Dobar dan. Daćete mi jednu kiflu. Koliko košta? Izvolite. Doviđenja. (etwa: 'Guten Tag. Wollen Sie mir ein Hörnchen geben. Wie viel kostet das? hier bitte. Auf Wiedersehen'); Dobar dan. Da li mogu da dobijem jednu kiflu? Hvala Vam. (etwa: 'Guten Tag. Kann ich ein Hörnchen bekommen? Danke schön'); Dobar dan! Molim vas da mi spakujete jednu kiflu. (etwa: 'Guten Tag. Ich bitte Sie, mir ein Hörnchen einzupacken.'); Dobar dan, mogu li dobiti kiflu sa makom? (etwa: 'Guten Tag, kann ich ein Hörnchen mit Mohn bekommen?') und Dobro jutro, ja cu jednu kiflu i jogurt. (etwa: 'Guten Morgen, ich möchte ein Hörnchen und ein Joghurt').

${ }^{31}$ Es wurden nur vollständige Äußerungen gezählt, die nicht nach der Entschuldigung abbrachen.
} 
Beebe, Leslie M./Clark Cummings, Martha (1996/2006): "Natural speech act data versus written questionnaire data: How data collection method affects speech act performance". In: Gass, Susan/Neu, Joyce (eds.) (2006): Speech Acts Across Cultures. Berlin/New York, de Gruyter: 65-88.

Blum-Kulka, Shoshana/Olshtain, Elite (1984): "Requests and Apologies: A Cross-Cultural Study of Speech Act. Realization Patterns (CCSARP)". Applied Linguistics 5/3: 196-213.

Blum-Kulka, Shoshana/House, Juliane (1989): "Cross-cultural and Situational Variation in Requesting Behavior". In: Blum-Kulka, Shoshana/House, Juliane/Kasper, Gabriele (eds.): Cross-cultural pragmatics: Requests and Apologies. Norwood. NJ, Ablex: 123-154.

Brown, Penelope/Levinson, Stephen C. (1987/2008): Politeness. Some universals in language usage. 17. print. Cambridge: Cambridge University Press.

Bybee, Joan/Dahl, Östen (1989): "The Creation of Tense and Aspect Systems in the Languages of the World". Studies in Languages 13/1, 51-103.

Economidou-Kogetsidis, Maria (2010): "Cross-cultural and situational variation in requesting behaviour: Perceptions of social situations and strategic usage of request patterns". Journal of Pragmatics 42 (2010): 2262-2281.

Engel, Ulrich/Mrazović, Pavica (eds.) (1986): Kontrastive Grammatik DeutschSerbokroatisch. 2 Bde. München: Sagner.

Goddard, Cliff/Ye, Zhengdao (2014): "Ethopragmatics". In: Sharifian, Farzad (ed.) (2014): The Routledge Handbook of Language and Culture. London, Routledge: 66-83.

Goffman, Erving (1955): "On Face-work: An Analysis of Ritual Elements of Social Interaction". Psychiatry. Journal for the Study of Interpersonal Processes 18/3, 213-231. Reprinted in Goffman, Erving (2005): Interaction ritual. Essays in face-to-face-behavior. New Brunswick, N.J: Aldine Transaction.

Gries, Stefan T. (2013): "Data in Construction Grammar". In: Hoffmann, Thomas/Trousdale, Graeme (eds.): The Oxford Handbook of Construction Grammar. Oxford, OUP: 93-132.

Hentschel, Elke (1991): "Aspect versus particle: Contrasting German and Serbo-Croatian". Multilingua 10-1/2: 139-149.

Hentschel, Elke (2003): "Wenn Partikeln frech werden". In: Held, Gudrun (ed.): Partikeln und Höflichkeit. Bern etc.: 55-72.

Hentschel, Elke (2012): "All men become brothers. The use of kinship terms for non-related persons as a sign of respect or disrespect". Linguistik online 51, 1/2012: 29-42.

Hentschel, Elke (2013a): "Verschiedene Wege, verschiedene Ziele: mal, schon und wohl". Germanistische Mitteilungen 39/1 (2013): 63-78.

Hentschel, Elke (2013b): "Kako se naručuje kifla". In: Nedeljković, Danica/Vujčić, Nikola (eds.) (2013): Jezik i književnost s obe strane zida. Kragujevac, Filološko-umetnički fakultet: $77-90$.

Holtgraves, Thomas (2005): "Social Psychology, Cognitive Psychology, and Linguistic Politeness". Journal of Politeness Research 1/1: 73-94.

Kim, Wonsun/Guan, Xiaowen/Park, Hee Sun (2012): "Face and Facework: A Cross-Cultural Comparison of Managing Politeness Norms in the United States and Korea". International Journal of Communication 6 (2012): 1100-1118.

Krivonosov, Aleksej (1963): Die modalen Partikeln in der deutschen Gegenwartssprache. Dissertation, Humboldt-Universität Berlin. 
Lee, Yu-Cheng (2011): "Comparison of Politeness and Acceptability Perceptions of Request Strategies between Chinese Learners of English and Native English Speakers". Asian Social Science 1/8 (2011): 21-34.

Leech, Geoffrey (2014): The Pragmatics of Politeness. Oxford: Oxford University Press.

Locher, Miriam A. (2012): "Politeness research from past to future, with a special focus on the discursive approach". In: Fernandez Amaya, Lucía et al. (eds.) (2012): New Perspectives on (Im)Politeness and Interpersonal Communication. Cambridge, Cambridge Scholars Publishing: 36-60.

Màrquez Reiter, Rosina (2000): Linguistic Politeness in Britain and Uruguay. A Contrastive Study of Requests and Apologies. Amsterdam/Philadelphia: J. Benjamins.

Milosavljević, Bojana (2007): Forme učitvosti u srpskom jeziku. Beograd: Učiteljski fakultet, Univerzitet u Beogradu.

Mrazović, Pavica/Vukadinović, Zora (1990): Gramatika srpskohrvatskog jezika za strance. Novi Sad: Dobra Vest.

Nishijima, Yoshinori (2014): "Politeness in Sign Expressions: A Comparison of English, German, and Japanese". Intercultural Communication Studies XXIII/2 (2014): 110-123.

Ogiermann, Eva (2009): "Politeness and Indirectness across Cultures: A Comparison of English, German, Polish and Russian Requests". Journal of Politeness Research 5 (2009): 189-216.

Pizziconi, Barbara (2007): "The lexical mapping of politeness in British English and Japanese". Journal of Politeness Research 3/2: 207-241.

Reihl, Bruno (2009): Der feine Unterschied. Ein Handbuch für Deutsche in der Schweiz. St. Gallen/Zürich: Midas.

Saldago, Elizabeth Flores (2011): The Pragmatics of Requests and Apologies. Developmental Patterns of Mexican Students. Amserdam/Philadephia: Nenjamins.

Schlund, Katrin (2009): Genese uns Gebrauch von Höflichkeitsformen im Serbischen und Deutschen. Ein funktionales Modell der Höflichkeit und seiner Anwendung. München/Berlin: Sagner. (= Dissertation, Universität Heidelberg).

Schopenhauer, Arthur (1851): Parerga und Paralipomena: kleine philosophische Schriften. 1. Band. Berlin: Heyn.

Searle, John (1975): "Indirect speech acts". In: Cole, P./Morgan, J.L. (eds.): Syntax and Semantics, 3: Speech Acts. New York: Academic Press: 59-82.

Stokar, Christoph (2112): Der Schweizer Knigge. Was gilt heute? Zürich: Beobachter.

Thomas, Alexander (2006): "Die Bedeutung von Vorurteil und Stereotyp im interkulturellen Handeln". Interculture journal: Online Zeitschrift für interkulturelle Studien. 5/2 (2006): http://www.interculture-journal.com/index.php/icj/article/view/46/55 [15.07.2014].

Tages Anzeiger, 14.12.2012.

Warga, Muriel (2004): Pragmatische Entwicklng In der Fremdsprache. Der Sprechakt "Aufforderung" im Französischen. Tübingen etc: Narr/Francke/Atempo.

Watts, Richard J. (2003): Politeness. Cambridge: Cambridge University Press.

Watts, Richard J, (2005) (ed.): Politeness in Language. Studies in its history, theory and practice. 2., rev. and expanded ed. Berlin/New York: Mouton de Gruyter.

Weydt, Harald (1969): Abtönungspartikel. Die deutschen Modalwörter und ihre französischen Entsprechungen. Bad Homburg/Berlin/Zürich: Gehlen. 
Zhu, Jiang/ Bao, Yuxiao (2010): "The Pragmatic Comparison of Chinese and Western 'Politeness' in Cross-cultural Communication". Journal of Language Teaching and Research, 6/1 (2010): 848-851.

\section{Anhang}

Fragebogen (Ausschnitt mit den hier diskutierten Situationen)

Deutsch:

Bitte versetzen Sie sich in die nachfolgend beschriebenen Situationen: Was würden Sie in dem Moment jeweils sagen? Falls Sie im Alltag Dialekt sprechen, können Sie ihre Antworten auch gerne im Dialekt aufschreiben.

Herzlichen Dank im Voraus!

- Sie wollen in einer fremden Stadt nach dem Weg zum Bahnhof fragen. Sie sprechen einen älteren Herrn an, der Ihnen zufällig begegnet, und sagen: ...

- Sie sitzen mit Ihrer Familie beim Essen und brauchen das Salz, das am anderen Ende des Tisches steht. Sie bitten Ihren jüngeren Bruder Daniel darum, indem Sie sagen: ...

- Sie wollen sich beim Bäcker eine Brezel kaufen. Als Sie an die Reihe kommen, sagen Sie:

Sagen Sie mir noch etwas zu Ihrer Person? (Das ist natürlich freiwillig!)

Sie sind:

- männlich

- weiblich

- SchweizerIn

- DeutscheR, und zwar aus (Bundesland bitte unten eintragen)

- ÖsterreicherIn

- andere Nationalität (bitte unten eintragen)

- $18-25$

- $25-35$

- $>35$

Serbisch:

Molim Vas, zamislite sebe u sledećim situacijama. Šta biste rekli u datom trenutku?

Unapred hvala što ste pristali da učestvujete $u$ anketi!

- Nalazite se u nepoznatom gradu i tražite železničku stanicu/kolodvor. Kad ugledate jednog starijeg gospodina, priđete mu i kažete: ...

- Sedite s porodicom za ručkom i zatreba Vam so, koja se nalazi na drugom kraja stola pored Vašeg mlađeg brata Saše. Okrenete mu se i kažete: ...

- Otišli ste kod pekara da kupite kiflu. Kad dođete na red, kažete: ... 
Ako želite, recite nešto o sebi. Vi ste:

- žensko

- muško

- Srbin/Srpkinja

- Hrvat/Hrvatica

- drugog porekla (molim Vas, napišete dole pod "Other" kojeg)

Vi imate

- 18-25 godina

- 25-35 godina

$>35$ godina 